\title{
CMS standard model Higgs boson results
}

\author{
Pablo Garcia-Abia ${ }^{1, a}$ for the CMS Collaboration \\ ${ }^{1}$ CIEMAT, Basic Research Department, Avda. Complutense 40, E-28005 Madrid (Spain)
}

\begin{abstract}
In July 2012 CMS announced the discovery of a new boson with properties resembling those of the long-sought Higgs boson. The analysis of the proton-proton collision data recorded by the CMS detector at the LHC, corresponding to integrated luminosities of $5.1 \mathrm{fb}^{-1}$ at $\sqrt{s}=7 \mathrm{TeV}$ and $19.6 \mathrm{fb}^{-1}$ at $\sqrt{s}=8 \mathrm{TeV}$, confirm the Higgs-like nature of the new boson, with a signal strength associated with vector bosons and fermions consistent with the expectations for a standard model (SM) Higgs boson, and spin-parity clearly favouring the scalar nature of the new boson. In this note I review the updated results of the CMS experiment.
\end{abstract}

\section{A new boson}

Observation of a SM-like Higgs boson, with a mass around $125 \mathrm{GeV} / \mathrm{c}^{2}$, was reported by the ATLAS [1] and CMS [2] Collaborations in July 2012. To establish the nature of this new particle it becomes essential to observe its decay modes and to measure with high precision its mass, spin-parity and couplings to bosons and fermions. To achieve this goal, the experiments perform exclusive analyses sensitive to the Higgs boson decay modes and production mechanisms. Recent results of the CMS experiment include $\mathrm{H} \rightarrow \gamma \gamma, \mathrm{H} \rightarrow \mathrm{ZZ} \rightarrow 4 \ell$, $\mathrm{H} \rightarrow \mathrm{WW} \rightarrow 2 \ell 2 v, \quad \mathrm{WH} \rightarrow \mathrm{WWW} \rightarrow 3 \ell 3 v, \quad \mathrm{t} \overline{\mathrm{t}} \mathrm{H}$ with $\mathrm{H} \rightarrow \mathrm{b} \bar{b}$, and $\mathrm{WH} / \mathrm{ZH}$ with $\mathrm{H} \rightarrow \tau \tau$. The latest results, new at LCHP 2013, are from the final states $t \bar{t} H$ with $\mathrm{H} \rightarrow \gamma \gamma, \mathrm{H} \rightarrow \mathrm{ZZ} \rightarrow 2 \ell 2 v$ and $\mathrm{H} \rightarrow \mathrm{b} \bar{b}$.

\section{LHC and the CMS detector}

The rich Higgs boson physics programme of CMS is possible thanks to the excellent performance of the LHC collider, which has provided proton-proton collisions at high luminosity, ever increasing since startup, The integrated luminosity collected by CMS is $5.1 \mathrm{fb}^{-1}$ at a centre-ofmass energy, $\sqrt{s}$, of $7 \mathrm{TeV}$ and $19.6 \mathrm{fb}^{-1}$ at $8 \mathrm{TeV}$. At this high luminosity multiple collisions per beam-crossing occur. It is an experimental challenge to cope with the high pile-up, which requires robust reconstruction software.

The Compact Muon Solenoid [3] (CMS) is a large compact fast-electronics detector with about 80 million electronic channels capable of operating at a frequency of $40 \mathrm{MHz}$. It is embedded in a highly uniform $3.8 \mathrm{~T}$ magnetic field, which allows for precise $3 \mathrm{D}$ event reconstruction. The data acquisition system is based on a high-efficiency low-latency trigger system that brings the

\footnotetext{
ae-mail: pablo.garcia@ ciemat.es
}

$20 \mathrm{MHz}$ collision rate down to about $800 \mathrm{~Hz}$, almost insensitive to pile-up. After 3 years of operation the efficiency of all the subdetectors of CMS stands above $96 \%$.

Individual particles are reconstructed using the particle flow algorithm, which provides a high efficiency identification of leptons (e, $\mu$ and $\tau$ ) and photons, very good momentum, energy and angular resolution of isolated particles and jets, efficient tagging of b-quark jets, and good missing transverse energy (MET) resolution. In general, efficiencies and resolutions are determined from control data samples.

\section{Higgs boson physics}

The SM Higgs boson can be produced at LHC via various mechanisms, among which the most important is gluon fusion. The total cross section is about $20 \mathrm{pb}^{-1}$ for a Higgs boson mass, $m_{\mathrm{H}}$, of $125 \mathrm{GeV} / c^{2}$. The partial decay width of the Higgs boson depends on the mass of the decay products. CMS exploits a large number of exclusive decays of the Higgs boson to determine the nature of the new state. This section summarises the latest results in several of these exclusive analysis, the next section focusing on their combination.

\section{1 $\mathrm{H} \rightarrow \gamma \gamma$ and $\mathrm{H} \rightarrow \mathrm{Z} \gamma$}

One of the main channels in which the Higgs boson was observed for the first time is its decay in two photons [4], $\mathrm{H} \rightarrow \gamma \gamma$. These are events with two high- $p_{\mathrm{T}}$ isolated photons with a narrow mass distribution, $m_{\gamma \gamma}$. In contrast, the di-photon mass distribution for the background from SM processes is steeply falling. The background expectation is evaluated from a fit to the data with no reference to the simulations. Multivariate techniques (MVA) are used to perform $\gamma$ identification and vertex determination.

Two inclusive analyses are performed. The main one selects events using a MVA constructed from $\gamma$ shower 
shape, isolation, kinematic variables, and the $m_{\gamma \gamma}$ resolution. As a cross-check, a cut-based selection is performed applying cuts on the $\gamma$ pseudo-rapidity and the $\gamma$ conversion probability. The events are classified in 4 categories with different signal over background (S/B) and mass resolution. To gain sensitivity on the Higgs boson production modes, 5 exclusive analyses are done: 3 channels with either one electron, one muon or MET tagged in the event (sensitive to $\mathrm{VH}$ ), and 2 dijet categories (sensitive to $\mathrm{VBF}$ ).

The di-photon mass distribution, with each event weighted by the $S /(S+B)$ value of its category, is displayed in figure 1. The Higgs boson signal shows up at $m_{\gamma \gamma}=125.4 \pm 0.5$ (stat.) \pm 0.6 (syst.) $\mathrm{GeV} / \mathrm{c}^{2}$, where the first uncertainty is statistical and the second systematic.

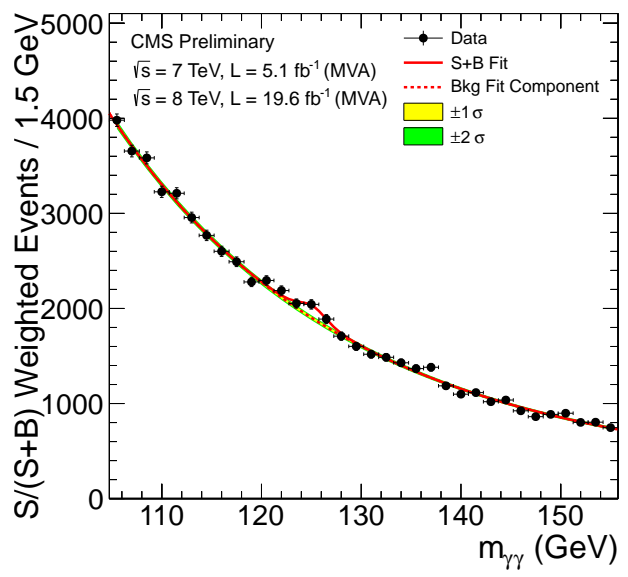

Figure 1. Diphoton invariant mass with each event weighted by the $S /(S+B)$ value of its category, for the MVA analysis on the 7 and $8 \mathrm{TeV}$ data sets combined. The lines represent the fitted background and signal, and the coloured bands are the \pm 1 and \pm 2 standard deviation uncertainties in the background estimate.

The exclusive analyses are combined using a profile likelihood ratio technique to evaluate the significance of the Higgs boson signal. This significance is usually expressed as the ratio of the production cross section times the relevant branching fractions over the SM expectation, $\sigma / \sigma_{\mathrm{SM}}$. In this analysis, $\sigma / \sigma_{\mathrm{SM}}=0.78 \pm 0.27$ for $m_{\mathrm{H}}=125 \mathrm{GeV} / c^{2}$, which can be expressed in terms of standard deviations $(\sigma)$ over the SM-background hypothesis (i.e. without a Higgs boson) as $3.2 \sigma$ observed and $4.2 \sigma$ expected for a SM Higgs boson. The significance of the signal is consistent among exclusive categories (figure 2).

The $\mathrm{H} \rightarrow \gamma \gamma$ decay is also observed in events in which the Higgs boson is produced in association with a $\bar{t} \bar{t}$ pair [5]. As a small signal is expected, two analyses, optimised for leptonic and hadronic $t \bar{t}$ decays, are performed to maximise the sensitivity. With the data available, a significant excess is not observed (figure 3) and upper limits at the $95 \%$ confidence level (CL) are set on $\sigma(\mathrm{t} \overline{\mathrm{t}} \mathrm{H}) \times \mathrm{BR}(\mathrm{H} \rightarrow \gamma \gamma)=5.4 \times \mathrm{SM}$, for $m_{\mathrm{H}}=125 \mathrm{GeV} / c^{2}$, where the expectation for a SM Higgs boson is 5.3.

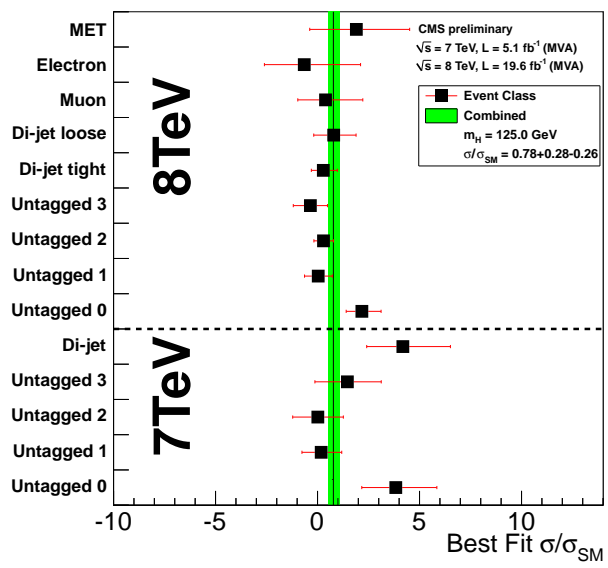

Figure 2. The best fit signal strength, $\sigma / \sigma_{\mathrm{SM}}$, obtained in the mass-fit-MVA analysis for the combined fit to the five classes used for the $7 \mathrm{TeV}$ data set and the nine classes used for the $8 \mathrm{TeV}$ data set (vertical line) and for the individual contributing classes (points) for the hypothesis of a SM Higgs boson mass of $125.0 \mathrm{GeV} / \mathrm{c}^{2}$. The band corresponds to $\pm 1 \sigma$ uncertainties on the overall value. The horizontal bars indicate $\pm 1 \sigma$ uncertainties on the values for individual classes.

The rare decay $\mathrm{H} \rightarrow \mathrm{Z} \gamma$ is also investigated [6]. Events with opposite-sign electron or muon pairs, from a $\mathrm{Z}$ boson, and a photon are selected. The signal expectation for this analysis is very low and events are split in several categories to improve $\mathrm{S} / \mathrm{B}$. There is no significant excess over the search region, in the $m_{\mathrm{H}}$ range from 120 to $150 \mathrm{GeV} / \mathrm{c}^{2}$. The observed exclusion limits at $95 \% \mathrm{CL}$ on $\sigma / \sigma_{\mathrm{SM}}$ are between 3 and 31, where expected between 6 and 19 .

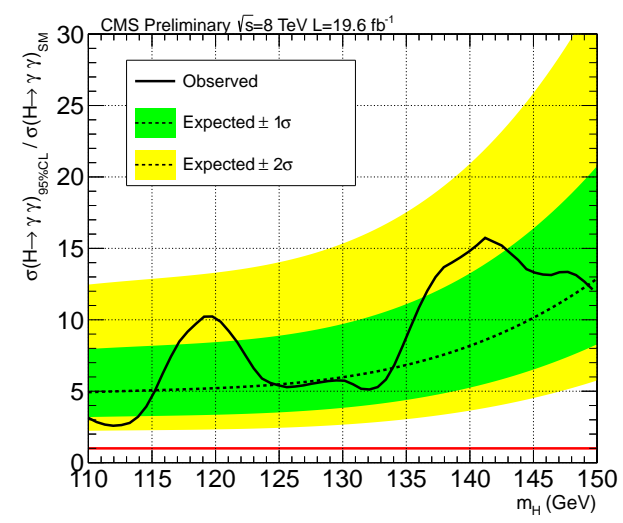

Figure 3. 95\% CL upper limit on $\mathrm{H} \rightarrow \gamma \gamma$ production combining the results of the $\bar{t} \bar{H} H$ leptonic and hadronic channels, divided by the SM production cross section times branching ratio.

\section{$3.2 \mathrm{H} \rightarrow \mathrm{ZZ} \rightarrow 4 \ell$}

The Higgs boson decay into 4 leptons (electrons or muons) is the golden channel in the first observation of the new boson [7]. Clean events with 4 high- $p_{\mathrm{T}}$ isolated leptons $(4 \mathrm{e}$, $4 \mu$ and $2 \mathrm{e} 2 \mu$ ) from the primary vertex, show up as a narrow 
mass resonance over a non-resonant background, mostly from ZZ SM processes (figure 4). Momentum resolution and scale are kept well under control using dedicated data samples. Mass resolution is measured from data. Due to the low branching ratio of this channel, it is important to keep lepton identification and selection efficiencies as high as possible. Two exclusive jet categories are considered in the analysis, untagged ( $0 / 1$ jets) and dijet tagged ( 2 or more jets), sensitive to different production modes. In the first category, the $p_{\mathrm{T}}(4 \ell) / m_{4 \ell}$ ratio is used to discriminate $\mathrm{VBF}$ and $\mathrm{VH}$ production from gluon-gluon fusion. In the second category a linear discriminant, formed from two VBF sensitive variables (the difference in pseudo-rapidity between the 2 leading jets and their invariant mass), is used to separate the VBF and gluon-gluon fusion processes.

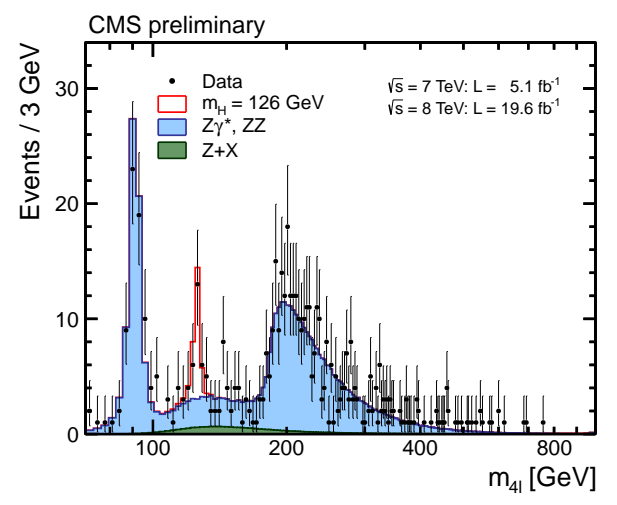

Figure 4. Four-lepton reconstructed mass in full mass range for the sum of the $4 \mathrm{e}, 4 \mu$, and $2 \mathrm{e} 2 \mu$ channels. Points represent the data, shaded histograms represent the background and unshaded histogram the signal expectations. The expected distributions are presented as stacked histograms.

A significant signal is observed in the 4-lepton mass distribution of figure 4 around $126 \mathrm{GeV} / c^{2}$. For mass values above $150 \mathrm{GeV} / \mathrm{c}^{2}$, data and the $\mathrm{SM}$ expectations agree, both in shape and normalisation, providing confidence in the background estimation. The radiative process $\mathrm{Z} \rightarrow 4 \ell$ is a reference candle at the $\mathrm{Z}$ boson mass, useful for calibration and background determination studies.

The kinematics of the $\mathrm{H} \rightarrow \mathrm{ZZ} \rightarrow 4 \ell$ events is fully reconstructed. The full matrix element of this process is expressed in terms of the Higgs and $\mathrm{Z}$ bosons decay angles in their respective rest frames. A linear discriminant, $K_{D}$, sensitive to the spin-parity of the particle decaying into $\mathrm{ZZ}$, is used to discriminate signal and background events. Figure 5 depicts the $2 \mathrm{D}$ distributions $K_{D}$ versus $m_{4 \ell}$ for data, for the expected background and for a $126 \mathrm{GeV} / \mathrm{c}^{2}$ Higgs boson signal. A concentration of events is visible at high values of $K_{D}$ for $m_{4 \ell}$ around $126 \mathrm{GeV} / \mathrm{c}^{2}$. To estimate the significance of the excess a $3 \mathrm{D}$ fit is performed to $m_{4 \ell}, K_{D}$ and, for the jet categories, either $p_{\mathrm{T}}(4 \ell) / m_{4 \ell}$ or the VBF linear discriminant. The best fit is obtained for $\sigma / \sigma_{\mathrm{SM}}=0.91_{-0.24}^{+0.30}$ at $m_{\mathrm{H}}=125.8 \pm 0.5$ (stat.) \pm 0.2 (syst.) $\mathrm{GeV} / c^{2}$, which corresponds to $6.7 \sigma$ observed, for a expectation of $7.2 \sigma$.
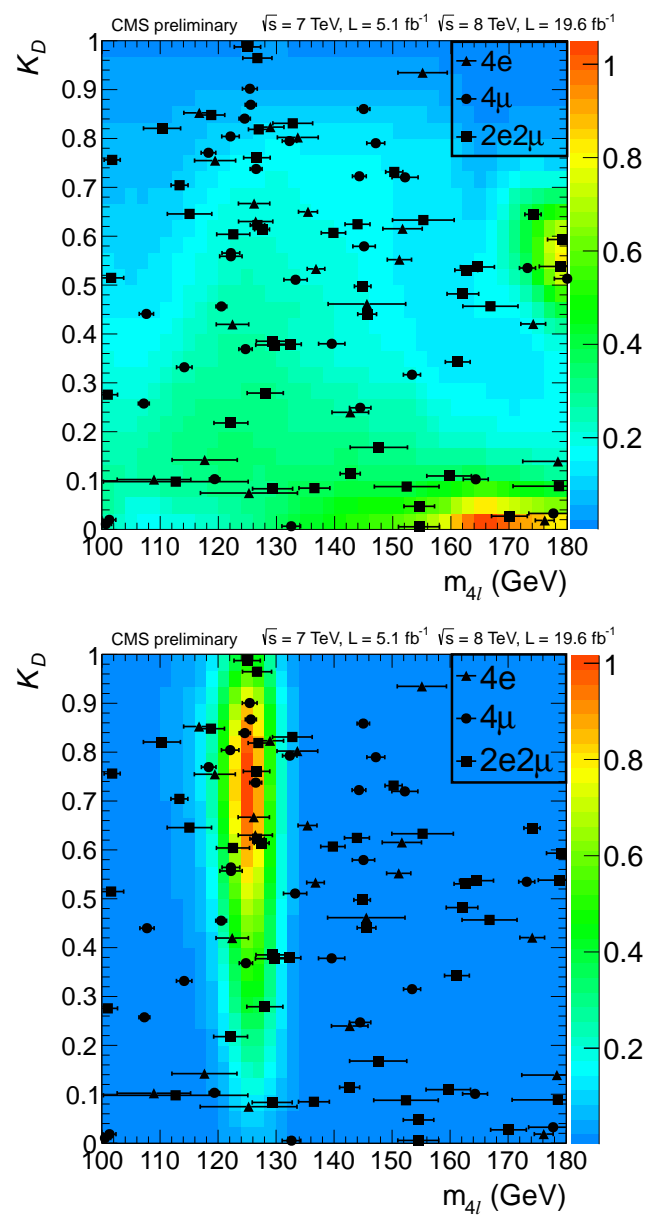

Figure 5. (Top) Kinematic discriminant $K_{D}$ versus the reconstructed mass, $m_{4 \ell}$. The points representing the individual events are shown together with their reconstructed mass uncertainties. The contours represent (top) the background expectation and (bottom) the signal expectation for $m_{\mathrm{H}}=126 \mathrm{GeV} / c^{2}$.

\section{$3.3 \mathrm{H} \rightarrow \mathrm{ZZ} \rightarrow 2 \ell 2 v$}

The $\mathrm{H} \rightarrow \mathrm{ZZ}$ channels play an important role also in the search for SM-like Higgs particles at high mass, above $200 \mathrm{GeV} / \mathrm{c}^{2}$. That is the case, in particular, for the $\mathrm{H} \rightarrow \mathrm{ZZ} \rightarrow 2 \ell 2 v$ process [8]. Clean events with 2 leptons $(\mathrm{e}, \mu)$ from the $\mathrm{Z}$ and large missing energy $(2 v)$ are selected in two analyses. Due to the presence of high- $p_{\mathrm{T}}$ neutrinos from the $\mathrm{Z}, m_{\mathrm{H}}$ is not reconstructed. The cut-in-categories and shape analyses are based on the transverse mass and missing energy. Jet categories are optimised separately for the VBF and ggH production mechanisms.

A SM-like Higgs boson is excluded by this analysis at the $95 \% \mathrm{CL}$ in the mass range from 248 to $930 \mathrm{GeV} / \mathrm{c}^{2}$ (figure 6). This result is interpreted in models beyond the SM (BSM), searching for an EW singlet scalar mixing with the new boson, which is excluded for various widths and branching ratios to new particles (details in [8]).

\section{$3.4 \mathrm{H} \rightarrow \mathrm{WW} \rightarrow 2 \ell 2 v$}

The observation of the Higgs boson benefits from the large branching ratio $\mathrm{H} \rightarrow \mathrm{WW} \rightarrow 2 \ell 2 v$ channel [9]. These 


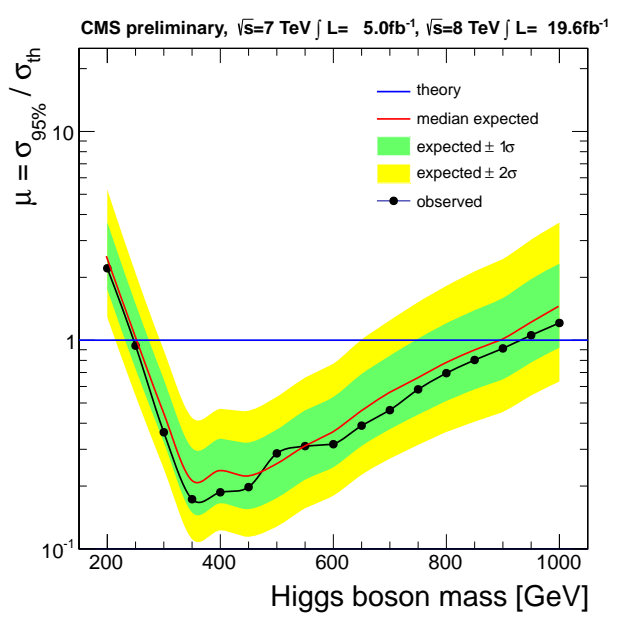

Figure 6. Upper limits on the signal strength obtained with the shape-based analysis in each event category using the combined dataset.The thick (dashed) lines show the evolution of the observed (expected) limits.

events have 2 high- $p_{\mathrm{T}}$ isolated leptons with low opening angle, driven by the spin configuration of the $\mathrm{H} \rightarrow \mathrm{WW}$ system. Due to the undetected neutrinos $m_{\mathrm{H}}$ is not reconstructed, hence the large MET is exploited in the analysis. The main background sources are Drell-Yan, WW, top and $\mathrm{W}+$ jets events, whose contribution is estimated from control regions in data. Contamination from $\mathrm{t} \overline{\mathrm{t}}$ events is rejected vetoing b-tagged jets. The events are split in 3 categories according to the number of jets $-0,1$ or 2 (VBF)-, gaining sensitivity on the production mechanism.

A significant excess of events is observed in the low mass region (figure 7), consistent with the expectation for a SM Higgs boson of $m_{\mathrm{H}}=125 \mathrm{GeV} / \mathrm{c}^{2}$. The cross section ratio is $\sigma / \sigma_{\mathrm{SM}}=0.76 \pm 0.21$, corresponding to $4 \sigma$ observed, to be compared to $5.1 \sigma$ expected for the SM Higgs boson at that mass. This result is consistent among the various analyses.

Both $\mathrm{H} \rightarrow \mathrm{ZZ} \rightarrow 4 \ell$ and $\mathrm{H} \rightarrow \mathrm{WW} \rightarrow 2 \ell v$ are sensitive to the spin-parity of the particle decaying into boson pairs. The $K_{D}$ linear discriminant is constructed in both analyses for different $J^{P}$ Higgs-like states having different kinematics, and used to compare various $J^{P}$ signal hypotheses. Our data significantly favour the $0^{+}$hypothesis, within $-0.34 \sigma$, as expected for a SM Higgs boson, disfavouring $0^{-}$and other spin 1 and 2 hypotheses. The $2_{\mathrm{m}}^{+}(\mathrm{gg})$ signal hypothesis $\left(2^{+}\right.$with minimal couplings to $\mathrm{gg}$ ) is excluded at $2.84 \sigma$.

\section{$3.5 \mathrm{WH} \rightarrow \mathrm{WWW} \rightarrow 3 \ell 3 v$}

Events with 3 high- $p_{\mathrm{T}}$ isolated leptons (e, $\mu$ ) large missing energy and low hadronic activity, are also used to observe the SM Higgs boson [10]. Background from WZ and top events is rejected applying a $\mathrm{Z}$ boson veto and anti-b-tagging. Two analyses, cut and shape based, exploit the smallest distance between opposite-charge leptons. No significant excess of events is observed and upper limits at

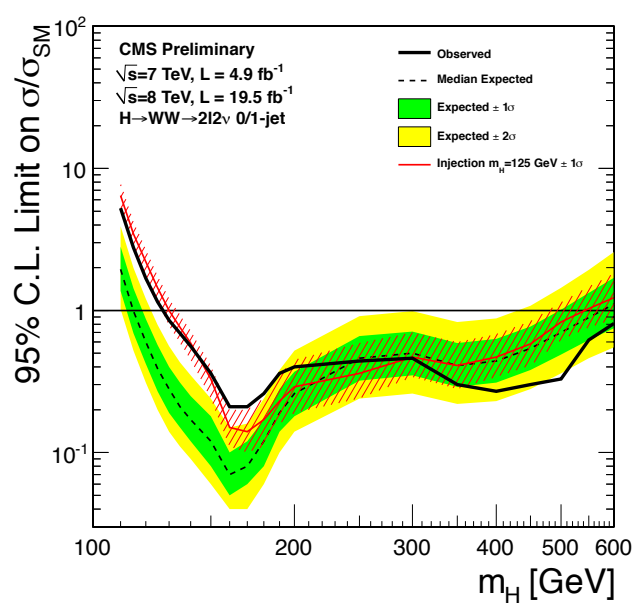

Figure 7. Expected and observed 95\% CL upper limits on $\sigma / \sigma_{\mathrm{SM}}$ for the shape-based approach using the 7 and $8 \mathrm{TeV}$ data. For the shape-based approach, we combine the analysis in the different-flavor final state in the 0 -jet and 1 -jet categories with the cut-based analysis in all other categories. The expected limits in the presence of the Higgs with $m_{\mathrm{H}}=125 \mathrm{GeV} / c^{2}$ and its associated uncertainty are also shown.

the $95 \% \mathrm{CL}$ are set on $\sigma / \sigma_{\mathrm{SM}}, 3.3$ observed and 3 expected, for $m_{\mathrm{H}}=125 \mathrm{GeV} / c^{2}$.

\section{$3.6 \mathrm{H} \rightarrow \mathrm{b} \bar{b}$}

The observation of the fermionic decays of the Higgs boson plays a crucial role in stating the SM-like nature of the new particle. Two $\mathrm{H} \rightarrow \mathrm{b} \bar{b}$ decay channels are exploited in $\mathrm{CMS}, \mathrm{VH} \rightarrow \mathrm{b} \overline{\mathrm{b}}+\mathrm{X}$, where $\mathrm{V}$ is either a $\mathrm{W}$ or a $\mathrm{Z}$ boson, and the VBF Higgs production, $\mathrm{VV} \rightarrow \mathrm{H} \rightarrow \mathrm{b} \overline{\mathrm{b}}$.

In $\mathrm{VH} \rightarrow \mathrm{b} \bar{b}+\mathrm{X}[11]$, events are characterised by two central $b$ jets plus a vector boson decaying into leptons and/or neutrinos. The main background stems from $\mathrm{V}+$ jets, $\mathrm{VV}$, top $+\mathrm{X}$. A shape analysis is performed constructing a boosted decision tree (MVA) from b-tagging and the kinematics of the jets and the vector bosons.

A broad excess in the mass distribution (figure 8), dominated by the jet resolution, is found to be compatible with a Higgs boson signal at low mass, with a significance for $m_{\mathrm{H}}=125 \mathrm{GeV} / c^{2}$ of $2.1 \sigma$ observed and $2.1 \sigma$ expected, corresponding to $\sigma / \sigma_{\mathrm{SM}}=1.0 \pm 0.5$, consistent among analyses of the various sub-channels.

The VBF $\mathrm{H} \rightarrow \mathrm{b} \overline{\mathrm{b}}$ analysis selects the fully hadronic final state (b-quark jets) [12], which is dominated by a large QCD background. Signal sensitivity is increased splitting the sample in 4 categories. The dijet mass distribution of b-tagged jets is used to discriminate signal from background. A regression technique improves the mass determination by $15 \%$ to $20 \%$. No significant excess of events is observed and 95\% CL upper limits are derived for five mass points from 115 to $135 \mathrm{GeV} / \mathrm{c}^{2}$. At a Higgs boson mass of $125 \mathrm{GeV} / \mathrm{c}^{2}$ the observed limit is 3.6 while the expected limit is 3.0 times the SM prediction, equivalent to a signal strength of $\sigma / \sigma_{\mathrm{SM}}=0.7 \pm 1.4$. 


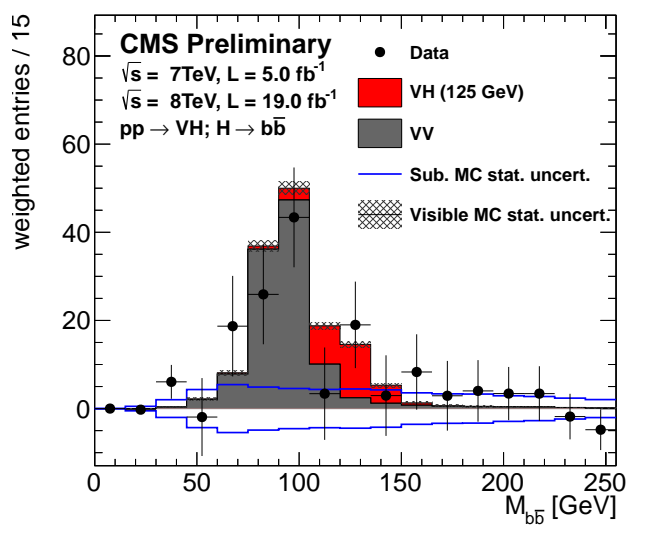

Figure 8. Weighted dijet invariant mass distribution with all backgrounds, except dibosons, subtracted, combined for all channels. The solid histograms for the backgrounds and the signal are summed cumulatively. The line histogram for signal and for VV backgrounds are also shown superimposed. The data is represented by points with error bars. The expected signal used corresponds to a $125 \mathrm{GeV} / \mathrm{c}^{2}$ Higgs boson.

\section{$3.7 \mathrm{H} \rightarrow \tau \tau$}

The Higgs boson decay into $\tau$ lepton pairs is an important evidence in favour of the SM-like nature of the new boson. $\mathrm{H} \rightarrow \tau \tau$ events are produced either via gluon fusion, or $\mathrm{WH} / \mathrm{ZH}$ or $\mathrm{VBF}[13,14]$. Several final states are analysed: $\mu \tau_{\mathrm{h}}$. e $\tau_{\mathrm{h}}, \mathrm{e} \mu, \tau_{\mathrm{h}} \tau_{\mathrm{h}}$ and $\mu \mu$. Isolated $\tau$ leptons hadronically decaying, $\tau_{\mathrm{h}}$, are identified using a MVA algorithm. The $\tau \tau$ mass is reconstructed using the SVFit algorithm, which exploits the kinematics of the $\tau$ decay, including the invariant mass of the $v v$ system in its leptonic decay, and using the transverse missing energy as a constraint. The improved mass reconstruction allows for a better discrimination between signal and the irreducible $\mathrm{Z} \rightarrow \tau \tau$ background. Additional contamination from other $\mathrm{SM}$ processes is due to $\mathrm{QCD}$ and $\mathrm{W}+$ jets.

Sensitivity on the production mode is achieved classifying events in categories according to the number of jets. The 0 -jet category, dominated by background, is useful to keep contamination under control. The 1-jet category is sensitive to the gluon fusion and $\mathrm{VH}$ production mechanisms, while the 2-jet category is most sensitive to VBF.

A broad excess in the $\tau$-pair mass distribution (figure 9) is found to be compatible with a $125 \mathrm{GeV} / \mathrm{c}^{2} \mathrm{Higgs}$ boson signal, with a significance observed of $2.89 \sigma$ and expected $2.6 \sigma$, that is $\sigma / \sigma_{\mathrm{SM}}=1.1 \pm 0.4$.

\section{Properties}

The combination of the Higgs boson searches requires simultaneous analysis of the data selected by all individual analyses, accounting for all statistical and systematic uncertainties and their correlations $[15,16]$. Updated results of the CMS combination can be found in [17].

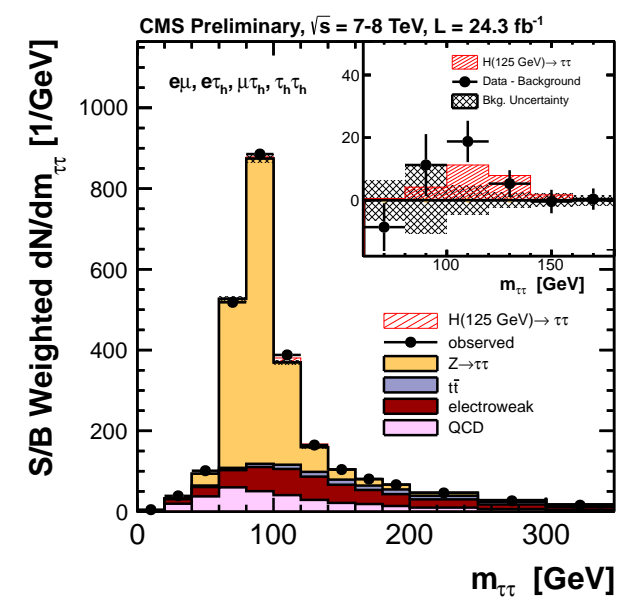

Figure 9. Combined observed and expected $m_{\tau} \tau$ distributions for the $\mu \tau_{\mathrm{h}}, \mathrm{e} \tau_{\mathrm{h}}, \mathrm{e} \mu$ and $\tau_{\mathrm{h}} \tau_{\mathrm{h}}$ channels. The distributions obtained in each category of each channel are weighted by the ratio between the expected signal and background yields in the category. The insert shows the corresponding difference between the observed data and expected background distributions, together with the expected signal distribution for a SM Higgs signal at $m_{\mathrm{H}}=125 \mathrm{GeV} / c^{2}$, with a focus on the signal region.

The mass of the observed state is measured from the two channels with excellent mass resolution, $\mathrm{H} \rightarrow \gamma \gamma$ (figure 1 ) and $\mathrm{H} \rightarrow \mathrm{ZZ} \rightarrow 4 \ell$ (figure 4 ). The 2D 68\% CL regions for the two parameters of interest, the signal strength modifier $\sigma / \sigma_{\mathrm{SM}}$ and the mass $m_{X}$, are shown in figure 10 for these channels. The combined $68 \% \mathrm{CL}$ contour is calculated assuming the SM Higgs boson relative event yield between the two channels, leaving the overall signal strength as a free parameter. The mass measurement, obtained in the combination of all search channels, is $m_{X}=125.7 \pm 0.3$ (stat.) \pm 0.3 (sys.) $\mathrm{GeV} / c^{2}$, and the best fit value for the common signal strength modifier $\sigma / \sigma_{\mathrm{SM}}=0.80 \pm 0.14$

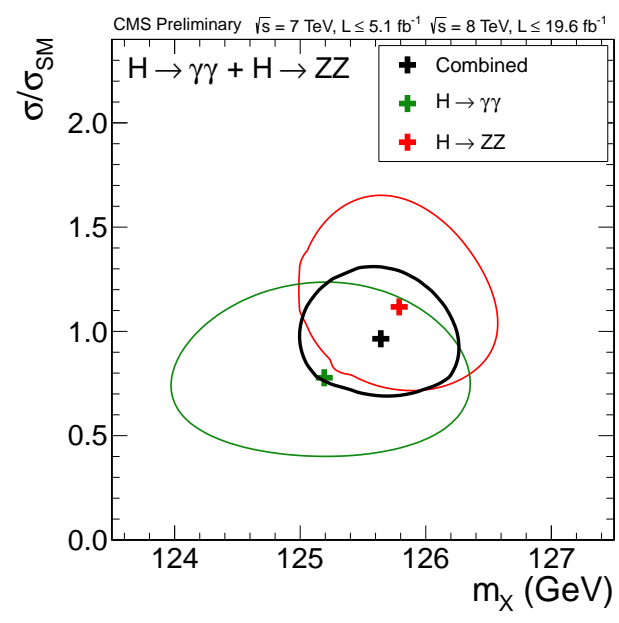

Figure 10. 2D 68\% CL contours for a hypothesised Higgs boson mass $m_{\mathrm{H}}$ and signal strength $\sigma / \sigma_{\mathrm{SM}}$ for the $\gamma \gamma$ and $4 \ell$ channels and their combination. 


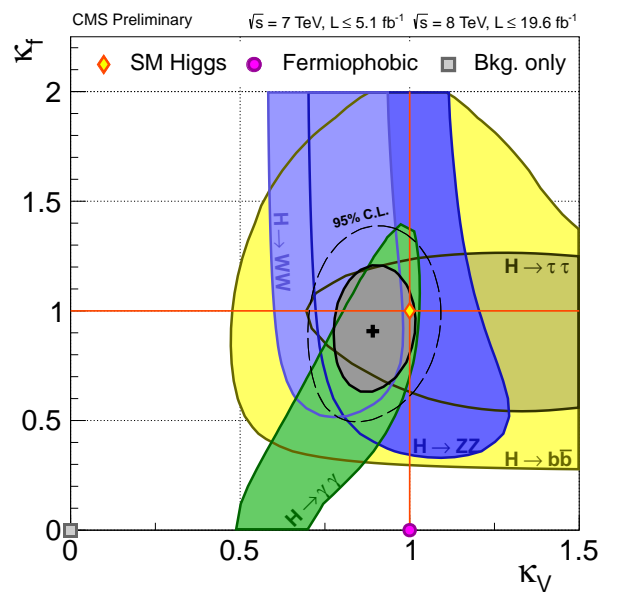

Figure 11. 2D likelihood scan showing the $68 \%$ CL contours for individual channels (coloured swaths) and for the overall combination (solid line) for the $\left(\kappa_{\mathrm{V}}, \kappa_{\mathrm{f}}\right)$ parameters. The cross indicates the global best-fit values. The thin contour shows the 95\% CL range for the combination. The yellow diamond shows the SM point $\left(\kappa_{\mathrm{V}}, \kappa_{\mathrm{f}}\right)=(1,1)$.

The event yield in any (production) $\times$ (decay) mode can be related to the production cross-section, and the partial and total Higgs boson decay widths:

$$
(\sigma \cdot \mathrm{BR})(x \rightarrow \mathrm{H} \rightarrow f f)=\frac{\sigma_{x} \cdot \Gamma_{f f}}{\Gamma_{t o t}}
$$

where $x$ represents the production mechanisms $(\mathrm{ggH}$, $\mathrm{VBF}, \mathrm{WH}$ and $\mathrm{ZH}$, and $\overline{\mathrm{t}} \mathrm{H}), \Gamma_{f f}$ is the partial decay width into the final state $f f(\mathrm{~W}, \mathrm{Z}, \mathrm{b}, \mathrm{t}, \gamma, \mathrm{Z} \gamma)$, and $\Gamma_{\text {tot }}$ is the total width of the Higgs boson. The size of the current dataset is insufficient to quantify meaningfully the phenomenological parameters defining the Higgs boson production and decay rates. Therefore, a few compatibility tests of the observed excesses with the expected signal are presented.

To test the custodial symmetry, the SM Higgs boson couplings to the $\mathrm{W}$ and $\mathrm{Z}$ bosons are modified introducing two scaling factors $\kappa_{\mathrm{W}}$ and $\kappa_{\mathrm{Z}}$ and two combinations are performed to verify the consistency of the ratio $\lambda_{\mathrm{WZ}}=\kappa_{\mathrm{W}} / \kappa_{\mathrm{Z}}=1$. From the fit to the data the $95 \% \mathrm{CL}$ interval for $\lambda_{\mathrm{WZ}}$ is $[0.62,1.19]$, consistent with the SM expectation.

Several tests of the couplings to the vector bosons and fermions, as well as tests for asymmetries in the couplings to fermions and of the presence for BSM particles, are performed. Figure 11 nicely summarises the deviations of the Higgs couplings to fermions, $\kappa_{\mathrm{f}}$, and bosons, $\kappa_{\mathrm{V}}$, with respect to the SM couplings. In all cases, excellent agreement is observed with the SM predictions.

\section{Conclusions}

The CMS Collaboration covered a challenging physics program in 3 years. To a large extent, it was possible thanks to the impressive performance of the LHC, the CMS detector and the computing system (Grid).

The observation of a new boson, reported last year, is confirmed with the latest data and additional channels investigated by CMS. The significance of most channels has increased with respect to ICHEP 2012. The significance of the VBF and VH processes is currently above $2 \sigma$. These results support the SM-like nature of the new boson.

A new era of precision measurements of the boson properties is ahead of us. Starting in 2015, a richer Higgs boson physics programme will include additional decay channels and new searches beyond the standard model.

\section{References}

[1] ATLAS Collaboration, Phys. Lett. B 716 (2012) 1-29, doi:10.1016/j.physletb.2012.08.020, arXiv:1207.7214.

[2] CMS Collaboration, Phys. Lett. B 716 (2012) 30-61, doi:10.1016/j.physletb.2012.08.021, arXiv:1207.7235.

[3] CMS Collaboration, JINST 3 (2008) S08004, doi:10.1088/1748-0221/3/08/S08004.

[4] CMS Collaboration, CMS-PAS-HIG-13-001 (2013), http://cds.cern.ch/record/1530524.

[5] CMS Collaboration, CMS-PAS-HIG-13-015 (2013), http://cds.cern.ch/record/1547292.

[6] CMS Collaboration, CMS-PAS-HIG-13-006 (2013), http://cds.cern.ch/record/1523674.

[7] CMS Collaboration, CMS-PAS-HIG-13-002 (2013), http://cds.cern.ch/record/1523767.

[8] CMS Collaboration, CMS-PAS-HIG-13-014 (2013), http://cds.cern.ch/record/1546776.

[9] CMS Collaboration, CMS-PAS-HIG-13-003 (2013), http://cds.cern.ch/record/1523673.

[10] CMS Collaboration, CMS-PAS-HIG-13-009 (2013), http://cds.cern.ch/record/1523681.

[11] CMS Collaboration, CMS-PAS-HIG-13-012 (2013), http://cds.cern.ch/record/1546801.

[12] CMS Collaboration, CMS-PAS-HIG-13-011 (2013), http://cds.cern.ch/record/1547579.

[13] CMS Collaboration, CMS-PAS-HIG-12-053 (2012), http://cds.cern.ch/record/1528147.

[14] CMS Collaboration, CMS-PAS-HIG-13-004 (2013), http://cds.cern.ch/record/1528271.

[15] ATLAS and CMS Collaborations, LHC Higgs Combination Group, Technical Report ATL-PHYS-PUB 2011-11, CMS NOTE 2011/005, (2011).

[16] CMS Collaboration, doi:10.1016/j.physletb.2012.02.064, arXiv:1202.1488.

[17] CMS Collaboration, CMS-PAS-HIG-13-005 (2013), http://cds.cern.ch/record/1542387. 